\title{
STUDIES ON A NEW ANTIBIOTIC, ALBOCYCLINE. II
}

\author{
TAXONOMIC STUDIES ON ALBOCYCLINE-PRODUCING STRAINS
}

\author{
Tamotsu Furumai, Noboru Nagahama and Tomoharu Okuda \\ Microbial Chemistry Research Laboratory, \\ Tanabe Seiyaku Co., Lıd., Toda, Saitama
}

(Received for publication August 3, 1967)

\begin{abstract}
Three different Streptomyces producing a new antibiotic albocycline were examined in reference to their taxonomic characteristics. It was concluded that two of the strains, S. MCRL-0129 and S. MCRL-0356, are new species of Streptomyces and they were named $S$. brunneogriseus nov. sp. and $S$. roseocinereus nov. sp., respectively. The third strain, S. MCRL-0355, was recognized to be a variety of $S$. roseochromogenes and named $S$. roseochromogenes var. albocyclini var. nov.
\end{abstract}

In the previous paper ${ }^{1)}$, production, isolation and properties of a new antibiotic named albocycline was reported. Introduction of gas chromatographic technique to the fermentation metabolites of this Streptomyces made it easy to distinguish the albocycline-producing strains from others. Thus, three albocycline-producing Streptomyces were isolated in our laboratory from soils collected at various places throughout Japan.

The present paper concerns the taxonomic studies on these albocycline-producing strains, S. MCRL-0129, S. MCRL-0356 and S. MCRL-0355, which were named Streptomyces brunneogriseus, $S$. roseocitreus and $S$. roseochromogenes var. albocyclini respectively.

\section{Taxonomic Studies}

Three microorganisms producing albocycline, S. MCRL-0129, S. MCRL-0356 and S. MCRL-0355, were isolated from soil samples respectively collected at Matsushima, Miyagi Prefecture, Seta, Gumma Prefecture and Shiozu, Kagawa Prefecture. The taxonomic studies on these strains were carried out according to accepted methods of

Table 1. Morphology of albocycline-producing Streptomyces

\begin{tabular}{|c|c|}
\hline Strain & Morphology \\
\hline S. MCRL-0129 & $\begin{array}{l}\text { Aerial mycelium long, straight or slightly wavy, forming sporophores } \\
\text { with numerous spirals with } 2 \text { to } 5 \text { turns. } \\
\text { Spores oblong, } 0.9 \sim 1.0 \text { by } 1.3 \sim 1.6 \mu \text {, smooth with phalangioform } \\
\text { structure. (Figs. } 1,2 \text { ) }\end{array}$ \\
\hline S. MCRL-0356 & $\begin{array}{l}\text { Sporophores producing open spirals as side branches on sterile aerial } \\
\text { mycelium. } \\
\text { Spores oval, } 0.6 \text { by } 1.1 \mu \text {, spiny. (Figs. } 3,4 \text { ) }\end{array}$ \\
\hline S. MCRL-0355 & $\begin{array}{l}\text { Sporophores monopodially branched, producing irregular open spirals at } \\
\text { the end of long hyphae. } \\
\text { Spores cylindrical, } 0.4 \sim 0.5 \text { by } 1.0 \sim 1.2 \mu \text {, smooth with phalangioform } \\
\text { structure. (Figs. } 5,6 \text { ) }\end{array}$ \\
\hline
\end{tabular}


Table 2. Cultural characteristics of albocycline-producing Streptomyces*1)

\begin{tabular}{|c|c|c|c|c|}
\hline \multicolumn{2}{|c|}{ Medium*2) } & S. MCRL-0129 & S. MCRL-0356 & S. MCRL-0355 \\
\hline $\begin{array}{l}\text { Glucose } \\
\text { nitrate } \\
\text { agar }\end{array}$ & $\begin{array}{c}\mathrm{G} \\
\mathrm{R} \\
\mathrm{AM} \\
\mathrm{SP}\end{array}$ & $\begin{array}{l}\text { camel ( } 3 \text { ie) } \\
\text { amber }(3 \mathrm{nc}) \\
\text { powdery, white } \\
\text { maple ( } 4 \text { le) }\end{array}$ & $\begin{array}{l}\text { luggage tan ( } 4 \text { ne) } \\
\text { dark luggage tan ( } 4 \text { pq) } \\
\text { powdery, white, scant } \\
\text { colonial yellow ( } 2 \text { ga) }\end{array}$ & $\begin{array}{l}\text { cinnamon ( } 3 \mathrm{le}) \\
\text { dark luggage tan }(4 \mathrm{pq}) \\
\text { powdery, white } \\
\text { amber }(3 \mathrm{lc})\end{array}$ \\
\hline $\begin{array}{l}\text { Glycerol } \\
\text { nitrate } \\
\text { agar }\end{array}$ & $\begin{array}{c}\mathrm{G} \\
\mathrm{R} \\
\mathrm{AM} \\
\mathrm{SP}\end{array}$ & $\begin{array}{l}\text { amber }(3 \mathrm{nc}) \\
\text { amber }(3 \mathrm{nc}) \\
\text { powdery, white } \\
\text { maple ( } 4 \mathrm{le})\end{array}$ & $\begin{array}{l}\text { dark luggage tan }(4 \mathrm{pq}) \\
\text { russet orange ( } 4 \mathrm{nc}) \\
\text { powdery, white, scant } \\
\text { colonial yellow ( } 2 \mathrm{ga})\end{array}$ & $\begin{array}{l}\text { cclorless to bright yellow } \\
\quad(2 \text { na) } \\
\text { bright yellow ( } 3 \text { na) } \\
\text { powdery, white } \\
\text { none or bright gold ( } 2 \mathrm{nc})\end{array}$ \\
\hline $\begin{array}{l}\text { Sucrose } \\
\text { nitrate } \\
\text { agar }\end{array}$ & $\begin{array}{c}\mathrm{G} \\
\mathrm{R} \\
\mathrm{AM} \\
\mathrm{SP}\end{array}$ & $\begin{array}{l}\text { colorless } \\
\text { colorless } \\
\text { powdery, beaver }(41 \mathrm{i}) \\
\text { none }\end{array}$ & $\begin{array}{l}\text { dark luggage } \tan (4 \mathrm{pq}) \\
\text { russet orange ( } 4 \mathrm{pc}) \\
\text { powdery, light ivory } \\
(2 \mathrm{ca}) \text {, scant } \\
\text { light yellow ( } 1 \frac{1}{2} \text { ea) }\end{array}$ & $\begin{array}{l}\text { colorless to bright yellow } \\
\qquad(2 \mathrm{na}) \\
\text { bright gold ( } 2 \mathrm{nc}) \\
\text { powdery, white, later powder } \\
\text { rose ( } 6 \mathrm{ec}) \\
\text { none or honey gold ( } 2 \text { ic) }\end{array}$ \\
\hline $\begin{array}{l}\text { Glucose- } \\
\text { asparagine } \\
\text { agar }\end{array}$ & $\begin{array}{c}\mathrm{G} \\
\mathrm{R} \\
\mathrm{AM} \\
\mathrm{SP}\end{array}$ & $\begin{array}{l}\text { bamboo ( } 2 \mathrm{gc}) \\
\text { colonial yellow ( } 2 \mathrm{ga}) \\
\text { powdery, beige ( } 3 \mathrm{ge}) \\
\text { later slate tan ( } 2 \mathrm{ig}) \\
\text { bright maize ( } 3 \mathrm{la})\end{array}$ & $\begin{array}{l}\text { amber ( } 3 \text { pe) } \\
\text { dark luggage tan ( } 4 \mathrm{pq}) \\
\text { velvety, rose gray ( } 6 \mathrm{ge}) \\
\text { with white patchs } \\
\text { none to colonial yellow } \\
\text { ( } 2 \text { ga) }\end{array}$ & $\begin{array}{l}\text { colorless to bright yellow } \\
\qquad(2 \text { na) } \\
\text { russet orange ( } 4 \mathrm{pc}) \\
\text { powdery, rose gray ( } 6 \mathrm{ge}) \\
\text { none or light wheat ( } 2 \mathrm{ea})\end{array}$ \\
\hline $\begin{array}{l}\text { Glucose } \\
\text { CzAPEK's } \\
\text { solution }\end{array}$ & $\left|\begin{array}{c}G \\
A M \\
S P\end{array}\right|$ & $\begin{array}{l}\text { light ivory ( } 2 \text { ca) } \\
\text { none } \\
\text { light mustard tan ( } 2 \text { ie) }\end{array}$ & $\begin{array}{l}\text { colorless } \\
\text { none } \\
\text { none }\end{array}$ & $\begin{array}{l}\text { light ivory ( } 2 \mathrm{ca}) \\
\text { none } \\
\text { none }\end{array}$ \\
\hline $\begin{array}{l}\text { Calcium } \\
\text { malate } \\
\text { agar }\end{array}$ & $\begin{array}{c}\mathrm{G} \\
\mathrm{R} \\
\mathrm{AM} \\
\mathrm{SP}\end{array}$ & $\begin{array}{l}\text { colorless } \\
\text { colorless } \\
\text { powdery, white } \\
\text { none }\end{array}$ & $\begin{array}{l}\text { colorless } \\
\text { colorless } \\
\text { powdery, white, scant } \\
\text { none }\end{array}$ & $\begin{array}{l}\text { colorless } \\
\text { colorless } \\
\text { powdery, white } \\
\text { none }\end{array}$ \\
\hline Starch agar & $\left|\begin{array}{c}\mathrm{G} \\
\mathrm{R} \\
\mathrm{AM} \\
\mathrm{SP}\end{array}\right|$ & $\begin{array}{l}\text { light brown ( } 4 \mathrm{ng}) \\
\text { oak brown ( } 4 \mathrm{pi}) \\
\text { powdery, rose gray }(6 \mathrm{ge}) \text {, } \\
\text { later taupe brown ( } 5 \mathrm{li}) \\
\text { none }\end{array}$ & $\begin{array}{l}\text { colorless } \\
\text { colorless } \\
\text { velvety, beige brown }(3 \\
\text { ig), with white patchs } \\
\text { none }\end{array}$ & $\begin{array}{l}\text { colorless to honey gold ( } 2 \mathrm{ic}) \\
\text { mustard gold }(2 \mathrm{pe}) \\
\text { powdery, powder rose }(6 \mathrm{ec}) \\
\text { cream }\left(1 \frac{1}{2} \mathrm{ca}\right)\end{array}$ \\
\hline $\begin{array}{c}\text { Tyrosin } \\
\text { agar }\end{array}$ & $\left|\begin{array}{c}G \\
R \\
A M \\
S P\end{array}\right|$ & $\begin{array}{l}\text { dark brown ( } 4 \mathrm{pn}) \\
\text { dark brown ( } 4 \mathrm{pn}) \\
\text { powdery, white, scant } \\
\text { dark brown ( } 4 \mathrm{nl})\end{array}$ & $\begin{array}{l}\text { colorless } \\
\text { colorless } \\
\text { powdery, white, scant } \\
\text { none }\end{array}$ & $\begin{array}{l}\text { chestnut brown ( } 4 \mathrm{ni}) \\
\text { fawn ( } 4 \mathrm{ig}) \\
\text { powdery, white } \\
\text { dark brown ( } 3 \mathrm{pn})\end{array}$ \\
\hline $\begin{array}{l}\text { Nutrient } \\
\text { agar }\end{array}$ & $\left|\begin{array}{c}\mathrm{G} \\
\mathrm{AM} \\
\mathrm{SP}\end{array}\right|$ & $\begin{array}{l}\text { colorless to ivory tint } \\
\qquad(2 \mathrm{cb}) \\
\text { none } \\
\text { dark brown }(3 \mathrm{nl})\end{array}$ & $\begin{array}{l}\text { yellow tint ( } 1 \mathrm{ba}) \\
\text { none } \\
\text { none }\end{array}$ & $\begin{array}{l}\text { yellow tint }(1 \mathrm{ba}) \\
\text { none } \\
\text { deep brown }(3 \mathrm{pl})\end{array}$ \\
\hline $\begin{array}{l}\text { Glucose } \\
\text { nutrient } \\
\text { agar }\end{array}$ & $\left|\begin{array}{c}\mathrm{G} \\
\mathrm{AM} \\
\mathrm{SP}\end{array}\right|$ & $\begin{array}{l}\text { ivory tint }(2 \mathrm{cb}) \\
\text { powdery, white } \\
\text { persimmon }(5 \mathrm{lc})\end{array}$ & $\begin{array}{l}\text { colorless } \\
\text { powdery, white, later } \\
\text { pearl ( } 3 \mathrm{ba}) \\
\text { light amber }(3 \mathrm{ie})\end{array}$ & $\begin{array}{l}\text { yellow tint ( } 1 \mathrm{ba}) \\
\text { powdery, white, scant } \\
\text { maple (4 le) }\end{array}$ \\
\hline
\end{tabular}


Table 2 (Continued)

\begin{tabular}{|c|c|c|c|c|}
\hline \multicolumn{2}{|c|}{ Medium $* 2)$} & S. MCRL-0129 & S. MCRL-0356 & S. MCRL-0355 \\
\hline $\begin{array}{l}\text { Glucose } \\
\text { peptone } \\
\text { agar }\end{array}$ & $\begin{array}{c}\mathrm{G} \\
\mathrm{AM} \\
\mathrm{SP}\end{array}$ & $\begin{array}{l}\text { light brown }(3 \mathrm{lg}) \\
\text { powdery, white, scant } \\
\text { dark brown }(3 \mathrm{nl})\end{array}$ & $\begin{array}{l}\text { colorless } \\
\text { powdery, white } \\
\text { none }\end{array}$ & $\begin{array}{l}\text { yellow tint ( } 1 \text { ba) } \\
\text { powdery, white } \\
\text { maple }(4 \mathrm{le})\end{array}$ \\
\hline $\begin{array}{l}\text { BENNETT'S } \\
\text { agar }\end{array}$ & $\left|\begin{array}{c}\mathrm{G} \\
\mathrm{R} \\
\mathrm{AM} \\
\mathrm{SP}\end{array}\right|$ & $\begin{array}{l}\text { light brown ( } 4 \mathrm{ng}) \\
\text { chestnut brown }(4 \mathrm{ni}) \\
\text { at first white, rose gray }(6 \\
\text { ge) to olive gray }(\mathrm{i} \text { ig), } \\
\text { later beige gray }(3 \mathrm{ih}) \\
\text { yellow maple }(3 \mathrm{ng})\end{array}$ & $\begin{array}{l}\text { colorless } \\
\text { dusty orange }(4 \mathrm{lc}) \\
\text { velvety, rose taupe }(5 \mathrm{ig}) \\
\text { with white patchs } \\
\text { none }\end{array}$ & $\begin{array}{l}\text { colorless } \\
\text { colorless } \\
\text { powdery, rose gray }(6 \mathrm{ge}) \\
\text { natural }(3 \mathrm{dc})\end{array}$ \\
\hline Potato plug & $\mid \begin{array}{c}\mathrm{G} \\
\mathrm{AM} \\
\mathrm{SP}\end{array}$ & $\begin{array}{l}\text { golden brown ( } 3 \mathrm{pi}) \\
\text { powdery, white, later } \\
\text { pearl ( } 3 \text { ba) } \\
\text { dark brown ( } 6 \mathrm{pn})\end{array}$ & $\begin{array}{l}\text { colorless } \\
\text { powdery, white to bright } \\
\text { yellow ( } 1 \frac{1}{2} \text { na) } \\
\text { none }\end{array}$ & $\begin{array}{l}\text { golden brown ( } 3 \mathrm{pi}) \\
\text { powdery, white, later pearl } \\
\quad(3 \mathrm{ba}) \\
\text { deep brown }(4 \mathrm{pl})\end{array}$ \\
\hline Egg medium & $\mid \begin{array}{c}\mathrm{G} \\
\mathrm{AM} \\
\mathrm{SP}\end{array}$ & $\begin{array}{l}\text { mustard } \tan (2 \mathrm{lg}) \\
\text { powdery, white } \\
\text { dark brown ( } 3 \mathrm{pn})\end{array}$ & $\begin{array}{l}\text { colorless } \\
\text { powdery, white to bright } \\
\text { yellow ( } 1 \frac{1}{2} \text { na) } \\
\text { none }\end{array}$ & $\begin{array}{l}\text { mustard tan }(2 \mathrm{lg}) \\
\text { powdery, white } \\
\text { dark brown }(3 \mathrm{pn})\end{array}$ \\
\hline Blood agar & $\left|\begin{array}{c}\mathrm{G} \\
\mathrm{AM} \\
\mathrm{SP}\end{array}\right|$ & $\begin{array}{l}\text { tile red ( } 5 \text { ne) } \\
\text { none } \\
\text { beige ( } 3 \mathrm{ge})\end{array}$ & $\begin{array}{l}\text { pale aqua green }(19 \mathrm{ca}) \\
\text { none } \\
\text { none }\end{array}$ & $\begin{array}{l}\text { cork } \tan (4 \mathrm{ie}) \\
\text { none } \\
\text { deep brown }(3 \mathrm{pl})\end{array}$ \\
\hline $\begin{array}{l}\text { Coagulated } \\
\text { serum }\end{array}$ & $\mid \begin{array}{c}G \\
\mathrm{AM} \\
\mathrm{SP}\end{array}$ & $\begin{array}{l}\text { camel ( } 3 \text { ie) to cinnamon } \\
\text { brown ( } 3 \mathrm{lg}) \\
\text { none } \\
\text { clove brown }(3 \mathrm{ni})\end{array}$ & $\begin{array}{l}\text { colorless } \\
\text { powdery, white, scant } \\
\text { none }\end{array}$ & $\begin{array}{l}\text { none } \\
\text { none } \\
\text { none }\end{array}$ \\
\hline Gelatin stab & $\left|\begin{array}{c}\mathrm{G} \\
\mathrm{AM} \\
\mathrm{SP}\end{array}\right|$ & $\begin{array}{l}\text { colorless } \\
\text { powdery, beige brown } \\
\qquad(3 \mathrm{ig}) \\
\text { dark brown }(3 \mathrm{nl})\end{array}$ & $\begin{array}{l}\text { colorless, scant } \\
\text { none } \\
\text { none }\end{array}$ & $\begin{array}{l}\text { beige brown ( } 3 \text { ig) } \\
\text { powdery, beige ( } 3 \text { ge) } \\
\text { dark brown ( } 3 \text { pn) }\end{array}$ \\
\hline Litmus milk & $\left|\begin{array}{c}G \\
A M \\
S P\end{array}\right|$ & $\begin{array}{l}\text { dark brown ( } 3 \mathrm{nl}) \\
\text { none } \\
\text { dark purple wine (11 pn) }\end{array}$ & $\begin{array}{l}\text { cream }\left(1 \frac{1}{2} \mathrm{ca}\right) \\
\text { none } \\
\text { none }\end{array}$ & $\begin{array}{l}\text { maple }(4 \mathrm{le}) \\
\text { none } \\
\text { dark purple wine (11 pn) }\end{array}$ \\
\hline Cellulose & $\begin{array}{c}G \\
A M \\
S P\end{array}$ & $\begin{array}{l}\text { light ivory ( } 2 \mathrm{ca}) \\
\text { none } \\
\text { biscuit }(2 \mathrm{ec})\end{array}$ & $\begin{array}{l}\text { colorless } \\
\text { none } \\
\text { none }\end{array}$ & $\begin{array}{l}\text { none } \\
\text { none } \\
\text { none }\end{array}$ \\
\hline
\end{tabular}

*1) Color names accord to Color Harmony Manual, 4th Ed. 1963. Container Corporation of America.

*2) G : growth, $\mathrm{R}$ : reverse, AM : aerial mycelium, SP : soluble pigment.

Fig. 1. Sporophores of $S$. Fig. 2. Electronmicrogram of MCRL-0129 on BENNETT's agar.

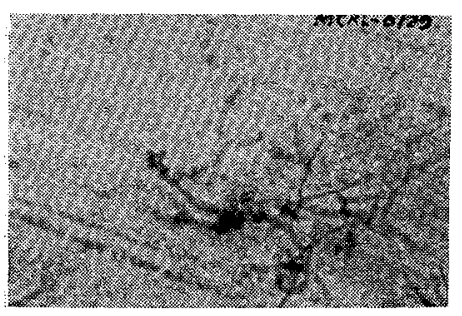
S. MCRL-0129 on BENNETT's agar. $(\times 5000)$

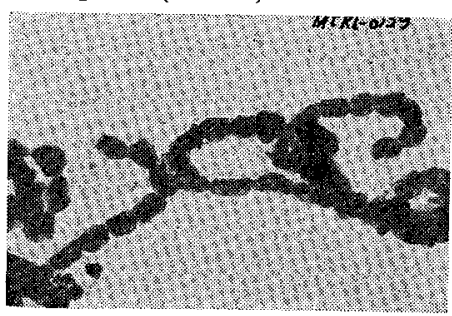

Fig. 3. Sporophores of $S$. MCRL-0356 on gulcoseasparagine agar.

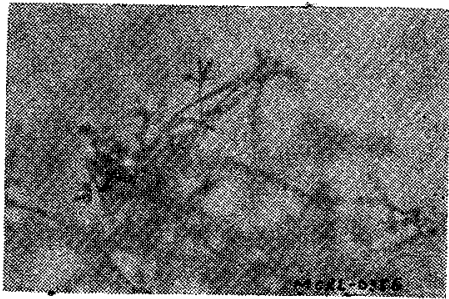


Fig. 4. Electronmicrogram of S. MCRL-0356 on BenNetT's agar. $(\times 6000)$

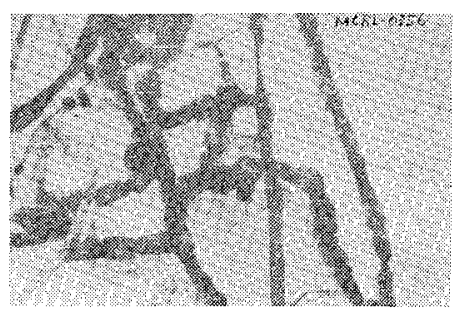

Fig. 5. Sporophores of $S$. MCRL-0355 on starch agar.

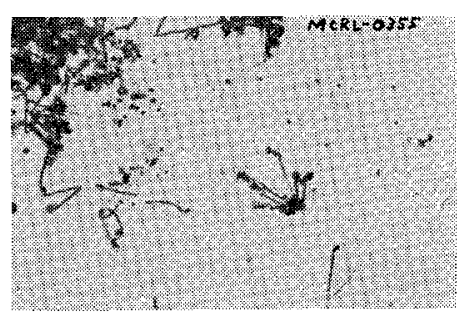

Fig. 6. Electronmicrogram of S. MCRL-0355 on starch agar. $(\times 6000)$

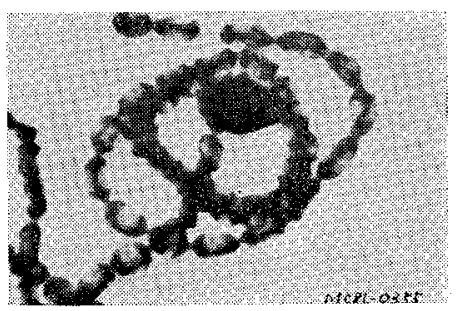

Table 3. Physiological characteristics of albocycline-producing strains

\begin{tabular}{l|l|l|l} 
& S. MCRL-0129 & S. MCRL-0356 & S. MCRL-0355 \\
\hline Solubilization of calcium malate & positive & negative & negative \\
Hydrolysis of starch & positive & weak & strong \\
Liquefaction of gelatin & strong & negative & negative \\
Hemolysis & positive & positive & weak \\
Milk coagulation & positive & positive & negative \\
Milk peptonization & positive & positive & positive \\
Milk reaction & acidic & acidic & acidic \\
Liquefaction of serum & negative & negative & negative \\
Reduction of nitrate & positive & negative & negative \\
Melanin formation & positive & negative & positive \\
$\mathrm{H}_{2}$ production & positive & negative & positive \\
Tyrosinase reaction & positive & negative & positive \\
Collulose decomposition & negative & negative & negative \\
\hline
\end{tabular}

identification for Streptomyces, particularly with regard to their taxonomic positions.

Morphology, cultural characteristics and physiological properties of these strains are illustrated in Tables 1,2 and 3 respectively. Utilization of carbohydrates by these strains tested according to PRIDHAM and GotTlieB ${ }^{2)}$ is summarized in Table 4. These strains grew well at $27^{\circ} \mathrm{C}$ as well as $37^{\circ} \mathrm{C}$ in the $\mathrm{pH}$ ranges of 5 to 9 , but they showed no growth at $5^{\circ}$ and $45^{\circ} \mathrm{C}$ in any $\mathrm{pH}$ ranges.

(1) S. MCRL-0129: S. MCRL-0129 grows colorless to pale yellowish on various agar media. In early stage aerial mycelium is white, but turns later to brownish white to brownish gray. Aerial mycelium on BENNETT's agar changes its color notably with time: brownish white
Table 4. Utilization of carbohydrates by albocycline-producing Streptomyces

\# : good utilization + : moderate utilization \pm : little utilization - : no utilization

\begin{tabular}{l|c|c|c}
\hline & S. & S. & S. \\
& MCRL-0129 & MCRL-0356 & MCRL-0355 \\
\hline Arabinose & + & + & + \\
Dextrin & \pm & + & + \\
Fructose & + & + & + \\
Galactose & + & + & + \\
Glucose & + & + & + \\
Glycerol & + & + & + \\
Inositol & - & + & + \\
Lactose & + & + & + \\
Maltose & + & + & + \\
Mannitol & + & + & - \\
Mannose & + & + & + \\
Raffinose & + & + & - \\
Rhamnose & - & + & - \\
Salicin & - & + & - \\
Starch & + & + & + \\
Sucrose & + & + & + \\
Xylose & + & + & + \\
Control & - & - & - \\
\hline
\end{tabular}


at first, then turning to grayish red, further to olive gray and finally to brownish gray. As shown in tables, this culture belongs to Spira-gray series according to the classification of PRIDHAM et al..$^{3,4)}$ and it is further characterized by producing phalangioform-spores ${ }^{5)}$ with smooth surface and by its chromogenic nature and strong proteolytic activity. Based on these characteristics, there is a suggestion that $S$. MCRL-0129 is related to $S$. aureus (WAKSMAN et CURTIS) WAKSMAN et HENRICI ${ }^{6}$, S. filipinensis AMMANN et al. ${ }^{7)}$, S. flavochromogenes (KRAINSKY) WAKSMAN et HENRICI ${ }^{8}$ and $S$. cinerochromogenes Mryairi et al. ${ }^{9)}$. The latest strain produces cineromycin $B$ which is quite similar to albocycline. However, S. aureus differs from S. MCRL0129 by dark brown growth and mouse gray aerial mycelium on CzapeK's agar, gray growth on nutrient agar, production of brown soluble pigment on gelatin stab, negative hemolytic activity and no utilization of arabinose. According to AMmANN et al..$^{7)}$ S. filipinensis grows best at $37^{\circ} \mathrm{C}$ and at $23 \sim 26^{\circ} \mathrm{C}$ it produces no aerial mycelium. It grows light yellowish, produces yellow soluble pigment on CzAPEK's agar, shows weak tyrosinase reactions, and little nitrate-reduction activity. These properties are different from those of S. MCRL-0129. S. flavochromogenes is differentiated from S. MGRL-0129 by its light brownish gray growth and production of brownish yellow soluble pigment on CzAPEk's agar, formation of gray aerial mycelium and brown soluble pigment on glucose-asparagine agar, yellow growth and gray aerial mycelium formation on calcium malate agar, and formation of spores without phalangioform structure. S. cinerochromogenes differs from S. MCRL-0129 in the respects that the former forms white aerial mycelium on CzAPEK's agar, produces no soluble pigment on glucose-asparagine agar, lacks ability to liquefy gelatin and to reduce nitrate, and hydrolyses starch slightly. S. cinerochromogenes and S. MCRL-0129 are also different in the utilization pattern of the following carbohydrates : inositol, mannitol, raffinose, rhamnose, and salicin.

In view of the above characteristics, S. MCRL-0129 was considered to be a new species and named Streptomyces brunneogriseus FuRUMAI et OKudA nov. sp. after the color of aerial mycelium on BENNETT's agar.

(2) S. MCRL-0356: The strain grows yellowish brown on synthetic agars. Spore formation was well observed on glucose-asparagine agar and starch agar. On these media the color of aerial mycelium was at first white, but, on maturity it turned beige brown to rose gray with white patches. It was noted that spores bear fairly long spine. The strain belongs to Spira-red series, and to non-chromogenic type. Proteolytic activity is rather weak.

The above properties suggested that S. MCRL-0356 is closely related to $S$. calvus BACKus et al. ${ }^{10)}$ However, S. calvus forms white, gray to mouse gray aerial mycelium on synthetic agars including glucose-asparagine agar and starch agar and never produces aerial mycelium of rose to reddish color. $S$. calvus has been reported to produce nucleocidin, but $S$. MCRL-0356 does not produce nucleocidin or any similar antibiotics. Thus, we concluded that S. MCRL-0356 is different from S. calvus, and should be assigned to a new species for which we propose the name of $S$. roseocinereus FuRUMAI et OKUdA nov. sp. based on the color of its aerial mycelium on starch agar 
or on glucose-asparagine agar.

(3) S. MRCL-0355: On synthetic agars, S. MCRL-0355 grows colorless to yellowish brown and produces aerial mycelium which is at first white and later turns to rose gray. Sucrose nitrate agar, starch agar, glucose-asparagine agar and BENNETT's agar are suitable for spore formation. Surface of spore is smooth and phalangioform. S. MCRL-0355 belongs to chromogenic type and Spira-red series. Proteolytic action of the strain is very limited.

It is suggested that $S$. MCRL-0355 is closely related to S. roseochromogenes (JENSEN) WAKSMAN and HHNRICI ${ }^{11)}$. The difference between both strains is small: $S$. roseochromogenes grows yellowish gray to brown-red and produces white to rose-gray aerial mycelium on nutrient agar and liquefies gelatin moderately. However, these differences are insufficient to assign S. MCRL-0355 to the rank of a new species of Streptomyces. Thus, we propose to call S. MGRL-0355 a variant strain of S. roseochromogenes and to name S. roseochromogenes (JENSEN) WAKSMAN and HENRICI var. albocyclini FURUMAI et OKUDA var. nov.

\section{Acknowledgement}

The authors wish to express their sincere thanks to Dr. Y. OKムMI and Dr. M. HAMADA, Institute of Microbial Chemistry, for the kind suggestions and advices.

\section{References}

1) Nagahama, N.; M. Suzuki, S. Awataguchi \& T. Okuda: Studies on a new antibiotic, albocycline. I. Isolation, purification and properties. J. Antibiotics, Ser. A 20(5) : 261 266, Sept. 1967

2) Pridham, T. G. \& D. Gotrlieb : The utilization of carbon compounds by some Actinomycetales as an aid for species determination. J. Bact. 56(1): 107 114, July 1948

3) Pridham, T.G.; P. Anderson, C. Folex, L. A. Lindenfelser, C. W. Hesseltine \& R.G. Benedict : A selection of media for maintenance and taxonomic study of Strefiomyces. Antibiot. Ann. 1956/1957, 947 953, 1957

4) Pridham, T. G.; C. W. Hesseltine \& R. G. Benedict : A guide for the classification of Streptomycetes according to selected groups. Placement of strains in morphological sections. Appl. Microbiol. 6(1):52 79, Jan. 1958

5) Tresner, H. D.; M. C. Davies \& M. E. EnGlert : Morphological subtype of the smooth-spored Streptomyces. J. Bact. 91(5) : 1998 2005, May 1966.

6) Waksman, S. A. : Cultural studies of species of Actinomyces. Soil Sci. 8(2):97 102, 1919

7) Ammann, A.; D. Gotplifeb, T. D. Brock, H. E. Carter \& G. B. Whitfield : Filipin, an antibiotic effective against fungi. Phytopathology $45: 559 \sim 563,1955$

8) Waksman, S. A. : Bergary's manual of determinative bacteriology. 6th. Ed. p. 941, 1948, The Williams \& Wilkins Co.

9) Miyatri, N.; M. Takashima, K. Shimizu \& H. SakaI : Studies on new antibiotics, cineromycins A and B. J. Antibiotics, Ser. A 19(2): 56 62, Mar. 1966.

10) Backus, E. J.; H. D. Tresner \& T. H. Campbell : The nucleocidin and alazopeptin producing organisms: Two new species of Streptomyces. Antibiot. \& Chemoth. 7(10):532 541, Oct. 1957

11) c. f. Waksman, S. A.: The actinomycetes. Vol. 2, p. 268 . The Williams \& Wilkins Co., 1961 